\title{
The genetics of phenotypic plasticity in adult abdominal colour pattern of Eristalis arbustorum (Diptera: Syrphidae)
}

\author{
MART M. OTTENHEIM* ${ }^{*}$, ANIQUE D. VOLMER $\dagger \&$ GRAHAM J. HOLLOWAY $\ddagger$ \\ $\dagger / n s t i t u t e$ of Evolutionary and Ecological Sciences, Section of Evolutionary Biology and Systematic Zoology, Leiden \\ University, PO Box 9516, 2300 RA, Leiden, The Netherlands and \$School of Animal and Microbial Sciences, AMS \\ Building, University of Reading, Whiteknights, PO Box 228, Reading RG6 2AJ, U.K.
}

\begin{abstract}
The abdominal colour pattern of Eristalis arbustorum is a plastic character which is heavily influenced by developmental temperature. The present study investigated the form of the reaction norms of amount of abdominal yellow and intensity of the yellow (measured as grey value) on pupal development temperature. The slope of the reaction norm for amount of yellow was steeper in males than in females. The reaction norms for grey value on temperature were nonlinear. Family groups were reared to enable a consideration of the genetics to be made. There were significant family by environment interactions for both characters for both sexes indicating genetic variance for plasticity. Pupal development time is closely correlated with developmental temperature. The relationship between amount of yellow on the abdomen and $\log$ pupal development time was curvilinear and fitted a quadratic function well. There was significant among-family variation in the slopes of these lines for females, but not for males, again suggesting genetic variation for plasticity. The results are discussed in relation to the maintenance of genetic variation.
\end{abstract}

Keywords: Eristalis, genetic variation, hoverflies, plasticity, reaction norm.

\section{Introduction}

Many organisms live in a seasonal or otherwise fluctuating environment and are regularly exposed to a variety of conditions. It often makes evolutionary sense for an organism to be able to adjust its phenotype to increase its fitness in relation to prevailing conditions. The ability of a single genotype to give rise to different phenotypes is referred to as phenotypic plasticity (Bradshaw, 1965). To study the evolution and genetics of plasticity, two models have been proposed: the 'character state' approach and the 'polynomial' approach (Via et al., 1995 and references therein). In the character state approach a character is modelled as a set of phenotypic values that would be expressed in each environment by a given genotype. Genetic variation for plasticity is demonstrated by a genotype by environment interaction (Via \& Lande, 1987). In the polynomial approach, a reaction norm is described by a polynomial function of the phenotypic values expressed

\footnotetext{
*Correspondence.
}

by a genotype across a range of environments. In this model genetic variation is demonstrated when the reaction norms cross. The models can be interchangeable under specific circumstances (Via et al., 1995).

The abdominal colour patterns of several hoverfly species show considerable plasticity (Heal, 1979a,b, 1981; Holloway, 1993). One of the best studied hoverflies in this respect is Eristalis arbustorum (L.) (Heal, 1981; Holloway, 1993; Ottenheim et al., 1995). When the pupa is kept at low temperatures a dark colour pattern develops and when it is kept at higher temperatures a lighter pattern develops (Heal, 1981; Ottenheim et al., 1995). As a consequence the colour pattern varies over the year with darker forms occurring in the cooler spring months and paler forms emerging during the warmer summer (Holloway, 1993). It has been argued that this kind plasticity may be adaptive and associated with the need for the insect to thermoregulate (Heal, 1981; Holloway, 1993).

The extent of the pale patterning on the abdomen of $E$. arbustorum is determined throughout the pupal 
stage and, indeed, pupal development time is strongly correlated with colour pattern size (Ottenheim et al., 1995). The wing patterns of many butterfly species (e.g. Pieris spp., Polygonia c-album, Bicyclus spp.) are also plastic characters (Nylin, 1989; Kingsolver \& Wiernasz, 1991; Windig, 1994). This plasticity is also often influenced by development temperature, particularly during the last larval stage and the first few days of pupal development (Nijhout, 1991). This results in some multivoltine species showing polyphenisms in the field, i.e. each generation exhibits a different wing colour pattern. However, these polyphenisms are generally determined by hormonal systems which, in their turn, are influenced by temperature or light regime (Koch, 1992). Considering these findings it is possible that E. arbustorum colour pattern is not influenced by temperature per se. It is probable that ambient temperature influences physiological processes during the pupal phase which determine the amount of yellow on the abdomen.

The purpose of this study was twofold. First, to determine the shapes of the reaction norms of two colour pattern characters on the abdomen of $E$. arbustorum. Secondly, to estimate the degree of significance of genetic variation for plasticity for the characters using both the 'character state' and 'polynomial' approaches described above.

\section{Materials and methods}

During April and May 1994, E. arbustorum adults were collected in and around Leiden (The Netherlands). Egg batches were collected from wild-caught female flies as described by Ottenheim \& Holloway (1995). Newly hatched larvae from each egg batch were initially reared at $20^{\circ} \mathrm{C}$ before being equally and randomly assigned to the experimental temperatures (see below). The larval rearing medium was based on rabbit droppings collected from a coastal dune system mixed with water. $20 \mathrm{~g}$ yeast (Saccharomyces cerivisiae siccum) was added to each litre of medium to reduce competition between larvae for food. The larvae were bred in small plastic trays $(18 \times 13 \times 4.5 \mathrm{~cm})$ which were separated into two equal compartments and could be closed with a transparent ventilated lid. In each tray between 14 and 20 larvae were reared in $200 \mathrm{~mL}$ medium.

To examine the effect of pupal development temperature on adult abdominal colour pattern flies were reared at six different temperatures: $8,11,14$, 17,20 and $26^{\circ} \mathrm{C}$ (all $\pm 1^{\circ} \mathrm{C}$ ). A light regime of $16 \mathrm{~h}$ light and $8 \mathrm{~h}$ dark was maintained throughout to ensure that at the lower temperatures the larvae would not go into diapause. For each family 20 larvae (one tray) were assigned at random to each temperature. Because a higher mortality was expected at $8^{\circ} \mathrm{C}$ (see Ottenheim et al., 1995) another 20 larvae were assigned to this temperature when family size allowed it.

The colour pattern of E. arbustorum is determined during the pupal stage (Ottenheim et al., 1995). Therefore the hatchling larvae were allowed to grow at $20^{\circ} \mathrm{C}$ for 11 days (i.e. developing into large third instar larvae) before being transferred to the different temperatures. Using this approach we were sure that all flies would experience the experimental temperatures throughout the whole of their pupal stage.

Pupae were collected daily from the larval medium and transferred to transparent Petri dishes which were kept in full light under the experimental temperature. Emergent adults were collected daily and were kept at $20^{\circ} \mathrm{C}$ for two days to ensure complete development of the abdominal colour pattern. After 2 days the flies were transferred to a $-20^{\circ} \mathrm{C}$ freezer for half a day after which they were dried and sexed.

The colour pattern on the abdomen of the adult flies was quantified using image analysis (see Ottenheim et al., 1995). The video image of a pinned fly was analysed using the T1M interactive image-analysis software. The flies were measured in several sessions. Before each session a size calibration was performed. A light intensity calibration was performed before and regularly during each session. A program was written to indicate natural boundaries on the image on the basis of contrast differences resulting in the segmentation of the colour pattern (for more details see Ottenheim et al., 1995).

The yellow/brown (hereafter referred to as yellow) colour pattern of E. arbustorum only appears on the second and third tergites. The characters measured were the area of yellow on the second tergite, the area of yellow on the third tergite, the area of black on the second tergite and the colour intensity (average grey value) of the yellow parts on the second tergite. From this the total area of yellow on the abdomen and the total area of the second tergite (a measure of abdomen size) were calculated. Because these two characters were phenotypically correlated $(r=0.771$ and 0.772 for males and females, respectively) the residuals of the regression of total yellow area on tergite size were used to analyse colour pattern size using analysis of variance.

To calculate a genotype by environment inter- 
action a two-factor analysis of variance was carried out using only those families which had representatives at all temperatures ( $\partial, 10$ families; $o, 9$ families). To determine the polynomial relation between pupal development time and colour pattern size, the method described by Sokal \& Rolf (1994) was followed. Pupal development time was natural logged to correct for non-normality. The overall relation between colour pattern size and pupal development time was significantly better explained by a quadratic function $\left(\hat{O}, R^{2}=63.1\right.$ per cent; + , $R^{2}=57.7$ per cent) than by a linear relationship ( $\hat{\sigma}$, $R^{2}=61.8$ per cent;,$+ R^{2}=55.5$ per cent) (s: $F_{1,486}=12.3, P<0.001$. $O_{0}: F_{1,514}=17.7, \quad P<0.001$ ). The linear component of the quadratic function made no significant contribution to the fit and was left out of the equation. Adding a cubed expression to the equations did not enlarge $R^{2}$ and was also omitted.

The relation between colour intensity and $\log$ development time for males was quadratic $\left(F_{1,486}=26.6, P<0.001\right)$, but for females an expression containing a cubed component explained significantly more of the observed variance than a linear or quadratic function $\left(F_{1,513}=10.2, \quad P<0.001\right)$. However, when the above relationship was analysed for each family separately, quadratic and cubed expressions showed nonsignificant fits to the data in all cases except for one family. Therefore, an ANCOVA could not be performed on the colour intensity data. The relation between colour pattern, size and pupal development time was significant for all families $(P<0.001$ in all cases $)$.

An ANCOVA was carried out on colour pattern size with family and squared log pupal development time as factors, and the latter also as a covariable. To analyse family differences in the relation between colour pattern size and pupal development time a analysis of covariance was performed using the GLM procedure in MINITAB 9.1.

\section{Results}

A total of 1005 flies was obtained of which 516 (51.3 per cent) were female. Overall survival was around 40 per cent. Survival was lower at 8 and $11^{\circ} \mathrm{C}$ than at the other temperatures.

Table 1 summarizes larval and pupal development times for males and females at the different temperatures. The results of the analyses are shown in Table 3. Both developmental periods were significantly shorter when the rearing temperature was higher. Larval development time did not vary between the sexes, but pupal development time did. Male pupae developed slightly, but significantly, faster than female pupae. For larval development time the interaction between sex and temperature was not significant, but for pupal development time this interaction was significant. The difference between male and female pupal development time decreased with increasing temperature.

The mean values of tergite (II) size, the total amount of yellow on tergites II and III, and the colour intensity for males and females for the various temperatures are summarized in Table 2 . Sex and the rearing temperature significantly influenced tergite size, but the response to temperature was the same for both sexes (Table $3, \mathrm{~S} \times \mathrm{T}=\mathrm{NS}$ ). Colour pattern size varied between the sexes. Males were on average more yellow than females and tended to be more responsive to temperature (Table $3, \mathrm{~S} \times \mathrm{T}=$ significant). Figure 1 shows the response of the colour pattern size to temperature for males and females. The colour intensity also varied between the sexes (Table 2, Fig. 2). The yellow on

Table 1 Development periods of male (§) and female (), larval (larval-D) and pupal (pupal-D) stages of Eristalis arbustorum at different rearing temperatures

\begin{tabular}{|c|c|c|c|c|c|c|}
\hline \multirow[b]{2}{*}{ Temp. } & \multicolumn{2}{|c|}{$n$} & \multicolumn{2}{|c|}{ Larval-D } & \multicolumn{2}{|c|}{ Pupal-D } \\
\hline & $\sigma^{2}$ & q & o & ㅇ & 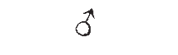 & q \\
\hline 8 & 55 & 61 & $26.2 \pm 0.91$ & $25.5 \pm 0.90$ & $37.6 \pm 1.07$ & $39.8 \pm 0.65$ \\
\hline 11 & 57 & 54 & $23.1 \pm 0.91$ & $22.8 \pm 0.90$ & $29.1 \pm 0.19$ & $29.6 \pm 0.17$ \\
\hline 14 & 104 & 101 & $18.3 \pm 0.42$ & $18.8 \pm 0.60$ & $15.4 \pm 0.13$ & $15.1 \pm 0.18$ \\
\hline 17 & 86 & 103 & $17.0 \pm 0.37$ & $17.6 \pm 0.32$ & $14.8 \pm 0.12$ & $14.9 \pm 0.10$ \\
\hline 20 & 103 & 108 & $15.5 \pm 0.32$ & $15.9 \pm 0.35$ & $8.1 \pm 0.10$ & $8.0 \pm 0.086$ \\
\hline 26 & 85 & 88 & $14.2 \pm 0.22$ & $14.3 \pm 0.23$ & $5.2 \pm 0.07$ & $5.2 \pm 0.088$ \\
\hline
\end{tabular}

Temperature in ${ }^{\circ} \mathrm{C}$; periods in days $\pm \mathrm{SE} ; n$, number of individuals. 
Table 2 Male (a) and female (b) Eristalis arbustorum average values for: size of tergite II $\left(\mathrm{mm}^{2} \pm \mathrm{SE}\right)$, colour pattern size (yellow, $\mathrm{mm}^{2} \pm \mathrm{SE}$ ) and colour intensity (intensity, average grey value \pm SE; white $=255$, black $=1$ ) for the different rearing temperatures (temp. ${ }^{\circ} \mathrm{C}$ )

\begin{tabular}{crlll}
\hline Temp. & $n$ & Tergite & Yellow & Intensity \\
\hline (a) & & & & \\
8 & 55 & $6.25 \pm 0.13$ & $2.32 \pm 0.096$ & $166 \pm 1.7$ \\
11 & 57 & $6.07 \pm 0.12$ & $2.51 \pm 0.095$ & $179 \pm 1.7$ \\
14 & 104 & $7.21 \pm 0.13$ & $4.73 \pm 0.11$ & $185 \pm 1.3$ \\
17 & 86 & $6.95 \pm 0.13$ & $4.42 \pm 0.11$ & $185 \pm 1.5$ \\
20 & 103 & $7.45 \pm 0.12$ & $5.66 \pm 0.11$ & $185 \pm 1.3$ \\
26 & 85 & $6.97 \pm 0.11$ & $5.44 \pm 0.11$ & $179 \pm 1.5$ \\
(b) & & & & \\
8 & 61 & $6.09 \pm 0.16$ & $1.26 \pm 0.11$ & $153 \pm 1.5$ \\
11 & 54 & $5.82 \pm 0.16$ & $1.34 \pm 0.11$ & $158 \pm 2.2$ \\
14 & 101 & $6.75 \pm 0.12$ & $2.74 \pm 0.083$ & $172 \pm 1.6$ \\
17 & 103 & $6.82 \pm 0.12$ & $2.86 \pm 0.072$ & $177 \pm 1.3$ \\
20 & 108 & $7.26 \pm 0.11$ & $3.71 \pm 0.077$ & $183 \pm 1.2$ \\
26 & 88 & $6.86 \pm 0.11$ & $3.57 \pm 0.077$ & $175 \pm 1.6$ \\
\hline
\end{tabular}

$n$, number of individuals.

Table 3 Analysis of variance $F$-values for larval (larval-D) and pupal (pupal-D) development times of Eristalis arbustorum, the size of the second tergite, the extent of yellow on the abdomen (colour pattern size) and the intensity of the yellow (measured in grey values) using the factors sex (d.f. 1, 994) and temperature (d.f. 5, 994)

\begin{tabular}{lccc}
\hline & Sex & Temp. & $\mathrm{S} \times \mathrm{T}$ \\
\hline Larval-D & $0.21^{\mathrm{NS}}$ & $127^{* * *}$ & $0.41^{\mathrm{NS}}$ \\
Pupal-D & $5.36^{*}$ & $3647^{* * *}$ & $4.33^{* *}$ \\
Tergite II & $9.16^{* *}$ & $31.1^{* * *}$ & $0.61^{\mathrm{NS}}$ \\
Colour pattern size & $2023^{* * *}$ & $60.2^{* * *}$ & $21.2^{* * *}$ \\
Colour intensity & $135^{* * *}$ & $439^{* * *}$ & $9.24^{* * *}$ \\
\hline
\end{tabular}

For the analysis of colour pattern size the amount of yellow on the abdomen was corrected for the size of tergite II (see text).

NS $=$ not significant $(P>0.05),{ }^{*} P<0.05,{ }^{* *} P<0.01$, ${ }^{* * *} P<0.001$

the second tergite was brighter for males than for females. At higher temperatures the yellow was also brighter than at lower temperatures. The lines for the two sexes have significantly different shapes, i.e. the interaction (Table $3, \mathrm{~S} \times \mathrm{T}$ ) is significant.

Within all temperatures there was significant variation among the families (Table 4) except at $11^{\circ} \mathrm{C}$ for both sexes and $26^{\circ} \mathrm{C}$ for the females. This indicates genetic variation for colour pattern size within most of the temperatures. A significant family by

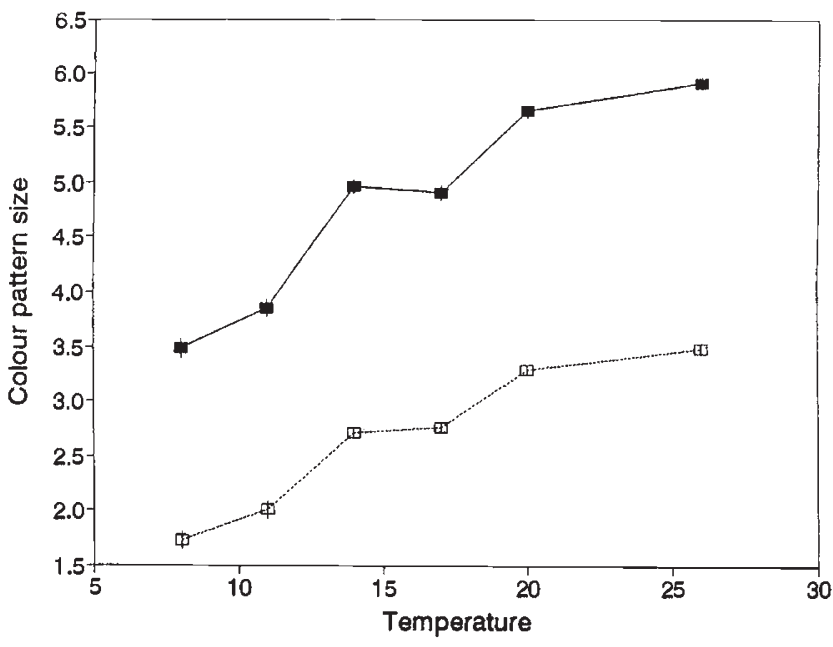

Fig. 1 The relation between colour pattern size and temperature for male ( $\square$ ) and female ( $\square$ ) Eristalis arbustorum. The bars indicate one standard error.

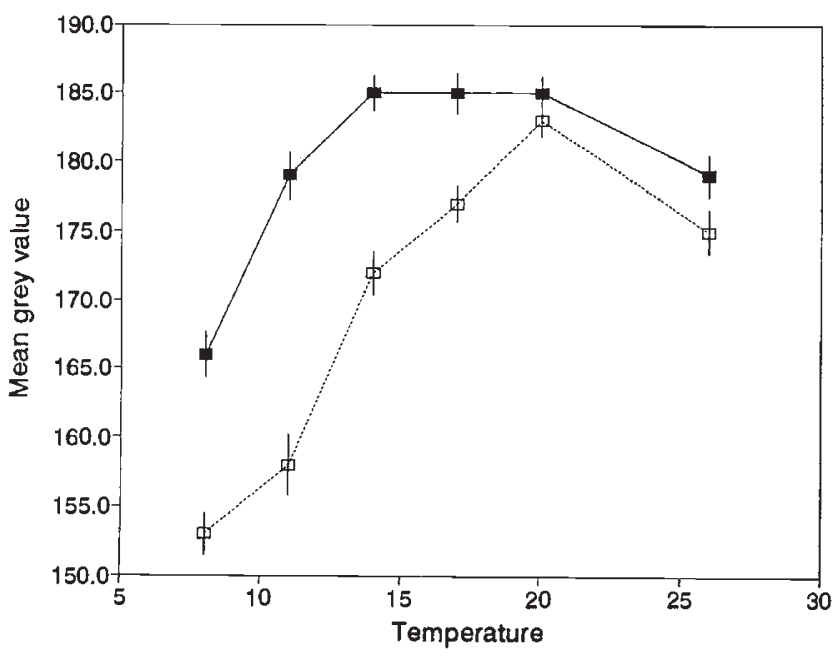

Fig. 2 The relation between colour intensity (grey value: $255=$ white, $1=$ black) and temperature for male $(\boldsymbol{a})$ and female ( $\square$ ) Eristalis arbustorum. The bars indicate one standard error.

environment interaction was found for both sexes for colour pattern size and for colour intensity (Table 5). The family reaction norms can been regarded as estimates of the reaction norms of single genotypes in a diploid organism. A significant family by environment interaction would then suggest a genotype by environment interaction indicating genetic variance for plasticity in amount of yellow and colour intensity.

Another way of testing for genetic variance for plasticity is to examine the slopes of the family reaction norms. Figure 3 shows the family estimations of 
the curvilinear relations between the extent of the colour pattern and the pupal development time. They differ significantly among the families for females $\left(F_{10,321}=2.95, P<0.001\right)$, but not for males $\left(F_{12,324}=1.24, P=0.254\right)$. This indicates the existence of genetic variance for plasticity of the colour pattern for females, but not for males.

\section{Discussion}

\section{Genetic variance}

In the E. arbustorum population used in this study there is genetic variance for plasticity in the amount of abdominal yellow and the intensity of the yellow. Several studies have demonstrated genetic variance for plasticity by means of genotype by environment

Table 4 Analysis of variance $F$-values for the extent of yellow on the abdomen of Eristalis arbustorum (colour pattern size) with family as factor within each temperature treatment

\begin{tabular}{lcccc}
\hline Temp. & Sex & d.f. & $F$ & $P$ \\
\hline 8 & m & 15,39 & 2.25 & $*$ \\
& f & 15,45 & 2.34 & $*$ \\
11 & m & 16,40 & 0.96 & NS \\
& f & 16,37 & 0.69 & NS \\
14 & m & 20,83 & 2.21 & $* *$ \\
& f & 18,82 & 4.00 & $* *$ \\
17 & m & 15,69 & 2.92 & $* * *$ \\
& f & 15,87 & 2.09 & $*$ \\
20 & m & 20,82 & 2.43 & $* *$ \\
& f & 19,88 & 6.10 & $* * *$ \\
26 & m & 16,68 & 4.98 & $* * *$ \\
& f & 16,70 & 1.74 & NS \\
\hline
\end{tabular}

For the analysis of colour pattern size the amount of yellow on the abdomen was corrected for the size of tergite II (see text).

NS $=$ not significant $(P>0.05), * P<0.05, * * P<0.01$, *** $P<0.001$. interactions (e.g. Zuberi \& Gale, 1976; Giesel et al., 1982; Rawson \& Hilbish, 1991; Windig, 1994) and others have used the method of variation among reaction norms (Windig, 1994). Both of these techniques have been used in the present study. The existence of genetic variance for plasticity might imply that it is possible for natural selection to act on the slope of the reaction norm enabling populations to adapt to local environmental conditions. However, there is not complete consensus about what this variance refers to (Via et al., 1995). Some consider plasticity to be a character with its own set of genes on which natural selection can act (Via et al., 1995 and references therein). But the genetic variance could also be the result of selection for environment-dependent character states and genetic uncoupling in different environments (Via et al., 1995).

Pupal development time is strongly correlated with temperature, which makes it difficult to establish which of the two is the proximate cue determining the colour pattern formation. Ottenheim et al. (1995) found a strong correlation between pupal development time and colour pattern size when development time was varied by transferring pupae between two temperatures. This implies that the colour pattern is responding to either development time or average temperature during the pupal period. In the present study we found that the mean pupal development times for $14^{\circ} \mathrm{C}$ and $17^{\circ} \mathrm{C}$ (Table 1 ) were not significantly different, and the extent of the colour pattern (Table 2) was also not different. This suggests that the extent of colour pattern may not be determined by temperature per se, but is more related to the development time of the pupa. One way to address this question might be to vary the pupal development time, perhaps using a selection regime, independent of the temperature.

Table 5 Analysis of variance $F$-values for amount of abdominal yellow and colour intensity for male (m) and female (f) Eristalis arbustorum separately, using the factors temperature (temp.) and family (fam.)

\begin{tabular}{|c|c|c|c|c|c|c|c|}
\hline & & \multicolumn{2}{|c|}{ Temp. } & \multicolumn{2}{|c|}{ Fam. } & \multicolumn{2}{|c|}{$\mathbf{T} \times \mathbf{F}$} \\
\hline & & d.f. & $F$ & d.f. & $F$ & d.f. & $F$ \\
\hline \multirow[t]{2}{*}{ Yellow } & $\mathrm{m}$ & 5,232 & $120.3^{* * *}$ & 9,232 & $8.06^{* * *}$ & 45,232 & $1.60^{*}$ \\
\hline & f & 5,243 & $57.4^{* * *}$ & 8,243 & $2.67^{* *}$ & 40,243 & $2.54^{* * *}$ \\
\hline \multirow[t]{2}{*}{ Intensity } & $\mathrm{m}$ & 5,232 & $12.4 * * *$ & 9,232 & $5.13^{* * *}$ & 45,232 & $2.66^{* * *}$ \\
\hline & f & 5,243 & $26.5^{* * *}$ & 8,243 & $4.50^{* * *}$ & 40,243 & $2.47^{* * *}$ \\
\hline
\end{tabular}

$* P<0.05, * * P<0.01, * * * P<0.001$. 

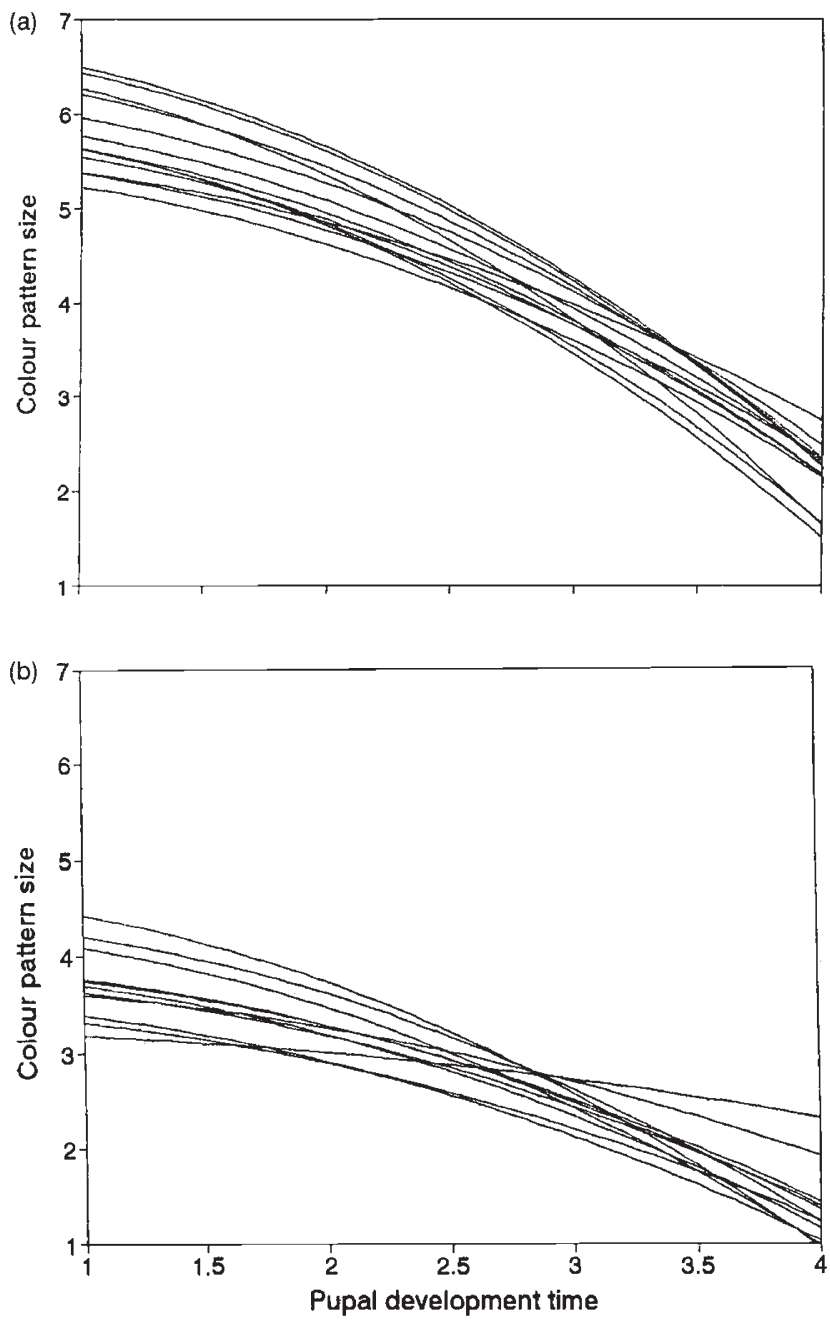

Fig. 3 The relation between colour pattern size and $\log _{e}$ pupal development time in different families for male (a) and female (b) Eristalis arbustorum.

\section{Linearity of the reaction norms}

Several studies of plasticity have examined character values in only two environments (e.g. Kingsolver \& Wiernasz, 1991; Ebert et al., 1993). Fewer studies have actually established the shape of reaction norms (Weiss \& Gorman, 1990; Rawson \& Hilbish, 1991; Windig, 1994; Holloway \& Brakefield, 1995). A knowledge of the form of the reaction norm is important as it may determine the most appropriate analysis to carry out. The relationship between colour pattern size and both temperature and $\log _{e}$ pupal period is not linear in E. arbustorum (Fig. 3). There appears to be an upper limit as to how large the colour pattern can be. Although the average extent of yellow did not differ significantly between the $20^{\circ}$ and $26^{\circ} \mathrm{C}$ treatments, some individuals had extensive areas of yellow on tergites II and III. A few females were obtained with small patches of yellow on the third tergite (M. M. Ottenheim, personal observation). As far as we know, this is not normally observed in field populations (Heal, 1981; Holloway, 1993).

The grey value (intensity) of the yellow on the abdomen is thought to be determined either just before or immediately after eclosion from the pupa (Ottenheim et al., 1995). Colour intensity also showed a limited response to temperature, especially in males (Fig. 2). The sexes responded differently to temperature, but this could have been an artifact of the measuring method. The colour tends to vary primarily in an anterior-posterior direction (Heal, 1981; Holloway, 1993). The colour intensity was measured only on the second tergite when possibly most variation in intensity in males occurred on the third tergite. On the other hand, significant sex-limited gene expression has been identified in other systems and it is possible that this colour-intensity sex effect, along with the obvious sexual dimorphism in abdominal pattern (see Fig. 1), is real. It is curious that the colour intensity is lower at $26^{\circ} \mathrm{C}$. We have, as yet, no explanation for this observation.

\section{Maintenance of genetic variance}

Natural selection tends to erode the genetic variance of a trait. We have demonstrated generally highly significant levels of genetic variance for the two components of the abdominal colour pattern in both sexes of E. arbustorum: the extent of the brownishyellow markings on the tergites and the brightness of this coloration. Modelling has shown that if natural selection can result in the evolution of an optimal reaction norm, the genotype by environment interaction contributes little to the maintenance of genetic variation (Via \& Lande, 1987). On the other hand, genotype by environment interactions could contribute significantly to the maintenance of genetic variation should an optimal reaction norm be unobtainable (Gillespie \& Turelli, 1989; Holloway \& Brakefield, 1995). The results of the present study are consistent with there being no optimal reaction norm possible for colour pattern in $E$. arbustorum. Alternatively, the environmental conditions fluctuate greatly from one year to the next opening up new, but short-lived, adaptive possibilities. A Red Queen equilibrium (van Valen, 1973) such as this could well contribute to the maintenance of genetic variation. A similar explanation has been forwarded to explain the high levels of genetic 
variation found in the polyphenic butterfly, Bicyclus anynana (Holloway \& Brakefield, 1995).

\section{Acknowledgements}

Jack Windig compiled the computer program for the image analysis for which we are very grateful. We are also grateful to G. Esmee Waller who helped with the breeding of the hoverflies. Jack Windig, Paul Brakefield and two anonymous referees made constructive remarks on the paper. The research reported here forms part of a Ph.D. project funded by the University of Leiden.

\section{References}

BRADSHAW, A. D. 1965. Evolutionary significance of phenotypic plasticity in plants. Adv. Genet., 13, 115-155.

EBERT, D., YAMPOLSKY, L. AND VAN NOORDWIJK, A. J. 1993. Genetics of life history in Daphnia magna. II. Phenotypic plasticity. Heredity, 70, 344-352.

GIESEL, J. T., MURPHY, P. A. AND MANLOVE, M. N. 1982. The influence of temperature on genetic interrelationships of life history traits in a population of Dosophila melanogaster: what tangled data sets we weave. Am. Nat., 119, 464-479.

GILLESPIE, J. H. AND TURELLI, M. 1989. Genotype-environment interactions and the maintenance of polygenic variation. Genetics, 121, 129-138.

HEAL, J. R. 1979a. Colour patterns of Syrphidae. I. Genetic variation in the dronefly Eristalis tenax. Heredity, 42, 223-236.

HEAL, J. R. 1979b. Colour patterns of Syrphidae. II. Eristalis intricarius. Heredity, 43, 229-238.

HEAL, J. R. 1981. Colour patterns of Syrphidae. III. Sexual dimorphism in Eristalis arbustorum. Ecol. Entomol., 6, 119-127.

HOLlowAY, G. J. 1993. Phenotypic variation in colour pattern and seasonal plasticity in Eristalis hoverflies (Diptera: Syrphidae). Ecol. Entomol., 18, 209-217.

HOLLOWAY, G. J. AND BRAKEFIELD, P. M. 1995. Artificial selection of reaction norms of wing pattern elements in Bicyclus anynana. Heredity, 74, 91-99.

косн, Р. в. 1992. Seasonal polyphenism in butterflies: a hormonally controlled phenomenon of pattern formation. Zool. Jb. (Physiol.), 96, 227-240.

KINGSOLVER, J. G. AND WIERNASZ, D. C. 1991. Development, function and the quantitative genetics of wing melanin pattern in Pieris butterflies. Evolution, 45, $1480-1492$.

Nishout, H. F. 1991. The Development and Evolution of Butterfly Wing Patterns. Smithsonian Institute Press, Washington and London.

NYLIN, s. 1989. Effects of changing photoperiods in the life cycle regulation of the comma butterfly, Polygonia c-album (Nymphalidae). Ecol. Entomol., 14, 209-218.

otTenheim, M. M. AND HOlloway, G. J. 1995. The effect of diet and light on larval and pupal development of laboratory reared Eristalis arbustorum (Diptera: Syrphidae). Neth. J. Zool., 45, 305-314.

OTTENHEIM, M. M., WALLER, G. E. AND HOLLOWAY, G. J. 1995. The influence of the development rates of immature stages of Eristalis arbustorum (Diptera; Syrphidae) on adult abdominal colour pattern. Physiol. Entomol., 20, 343-348.

RAWSON, P. D. AND HILBISH, T. H. 1991. Genotype-environment interaction for juvenile growth in the hard clam Mercenaria mercenaria (L.). Evolution, 45, 1924-1935.

SOKAL, R. R. AND ROLF, F. J. 1994. Biometry, 3rd edn. W. H. Freeman, New York.

VAN VALEN, L. M. 1973. A new evolutionary law. Evol. Theor, 1, 1-30.

VIA, S. AND LANDE, R. 1987. Evolution of genetic variability in a spatially heterogeneous environment: effects of genotype-environment interaction. Genet. Res., 49, $147-156$.

VIA, S., GOMULKIEWICZ, R., DE JONG, G., SCHEINER, S. M., SCHLICHTING, C. D. AND VAN TIENDEREN, P. H. 1995. Adaptive phenotypic plasticity: consensus and controversy. Trends Ecol. Evol., 10, 212-217.

WEISS, A. E. AND GORMAN, W. L. 1990. Measuring selection on reaction norms: an exploration of the Eurosta-Solidago system. Evolution, 44, 820-831.

wINDIG, J. J. 1994. Reaction norms and the genetic basis of phenotypic plasticity in the wing pattern of the butterfly Bicyclus anynana. J. Evol. Biol., 7, 665-695.

ZUBER1, M. 1. AND GALE, J. s. 1976. Variation in wild populations of Papaver dubium. X. Genotype-environment interaction associated with differences in soil. Heredity, 36, 359-368. 